\title{
El catastro de Ensenada y su proceso de formación (1750-1760)
}

\author{
Javier M. Donézar Díez de Ulzurrun *
}

La Unica Contribución propuesta por el marqués de la Ensenada era un impuesto sobre la renta en su doble modalidad: sobre los haberes y sobre el trabajo personal. En una economia agraria, como la del Antiguo Régimen, con un peculiar reparto de la propiedad la medida iba a ser altamente perjudicial para las clases cuya existencia estaba basada en la apropiación de los excedentes - las privilegiadas - y liberalizadora para los que, produciéndolos, no eran beneficiarios finales de los mismos.

Sin embargo, esta renta no era equivalente al produit net de Quesnay. Para éste, únicamente el sector agricola producia excedentes materiales porque contaba con el factor tierra, con el "don gratuito", que devolvía más cantidad de producto que el que se había sembrado. Quesnay creía, como Ensenada, que había que establecer el impuesto sobre la renta, pero sobre "su peculiar tipo de renta" - el de la tierra-, que implicaba que los demás sectores (manufactura y comercio) no la producian.

Ensenada tenía un concepto más avanzado de renta e intentó gravar todo beneficio y utilidad. El impuesto recaería sobre el destinatario de la renta, tuviera ésta la forma que fuese: de la tierra, de alquileres de casas, de réditos de censos o de juros, o de la percepción de impuestos enajenados.

* Catedrático de Historia Contemporanea de España de la Universidad de Castilla-La 
Suponia ello atacar a los estamentos privilegiados «improductivos", el 'beau monde' absentista — que mencionaba Boisguilbert-, y liberar a la clase "productiva» agrícola, arrendataria, que originaba esa renta; era incitar al "empresario" de Cantillon a crear riqueza, a aquel que se caracterizaba por tener unos gastos fijos y unos ingresos inciertos, que se movía de acuerdo con su interés individual y gracias a su inteligencia podía ir formando un capital.

No se intentaba, pues, con la Única, gravar la propiedad en cuanto tal sino la propiedad de la renta; la distinción, a primera vista, puede parecer ociosa. Pero si no, ¿cómo entender que grandes extensiones de tierras de particulares fueran reguladas por la cantidad de su arrendamiento, o que las extensiones de los pueblos aparecieran con «nula producción» o que a molinos y otros artefactos, cuando figuraban como inactivos, no se les asignase ninguna valoración? No era un impuesto sobre el bien inmueble sino sobre la renta de ese bien.

¿Por qué se operó, entonces, en la tierra con el producto bruto? Fue, más bien, una pretendida forma de proceder o, si se quiere, la única posible para llegar a saber la "verdadera riqueza". Lo que reflejan las Respuestas Particulares con respecto a las tierras cultivadas, y no otras, era el primer paso para acceder a la propiedad de la renta y, por tanto, no el último. Al propietario de la tierra se le exigía la declaración de sus parcelas y su contenido (extensión, calidad y dedicación) pero nada más, ni siquiera un cálculo de su producto bruto anual en dinero. Escribir éste al margen de cada parcela era labor de oficina de los empleados de acuerdo con los datos que obraban en su poder por el Interrogatorio General.

Siempre resultaría más difícil para el propietario eludir la existencia de una superficie, que era "visible para todos" que el producto logrado $y$, por supuesto, la renta. Luego, otras normas indicarian el modo de convertir dicho producto bruto en neto. En el apartado XII de la Instrucción de 1760 se decía:

«Para las utilidades que deben estimar y valuar los referidos peritos $y$ tasadores y efectos del ramo real, han de considerar los gastos y expensas en el diezmo de sus frutos y productos; en los ganados, el que tengan las yerbas, pastoreo de ellos y su diezmo; y en las casas, edificios y otros artefactos, lo que correspondan a huecos y reparos, arreglándose en la baja de los expresados gastos a las calidades de los efectos y circunstancias de los pueblos". 
Que el Proyecto estaba destinado a los propietarios de renta lo entendieron enseguida los futuros contribuyentes que se apresuraron a declarar menos - no bienes inmuebles sino producto, rebajando la calidad de la tierra o subiendo los gastos- o a incrementar los arrendamientos para paliar los efectos de aquél.

Donde si aparece una cierta confusión entre producto bruto y neto es en la regulación del trabajo personal. Figuraban los salarios como utilidades, como productos netos, al igual que las ganancias provenientes de los negocios, siendo como eran productos brutos que sólo después de haber efectuado con ellos los gastos pertinentes para la subsistencia podrian convertirse en netos. Pero esta confusión no invalida el plan de Ensenada: todavía faltaban años para que se perfilaran los conceptos de trabajo y salario en la economía. En el aún planteamiento mercantilista, el salario por trabajar a cuenta ajena era un líquido que añadía el individuo a su haber y un gasto deducible del producto bruto del que lo pagaba, y siempre un ahorro para el que trabajaba por su cuenta.

Poco se ha estudiado sobre el proceso de formación del Catastro, obra magna de los ilustrados, atentos los investigadores más a los resultados lo cual, por otra parte, resulta obvio. Pero tal empresa requería todo un esquema organizativo que no es posible pasar por alto.

En cada una de las provincias, la operación comenzó por la elección de un pueblo que reuniese los mayores requisitos posibles; sobre lo que en él se hiciese se irian dictando los procedimientos convenientes para los demás.

En el caso de la provincia de Toledo, que hemos estudiado, la villa elegida fue Ajofrín y según se comunicaba en carta de 18 de septiembre de 1750: «por considerarla V.S. con todos frutos y una fábrica de lanas

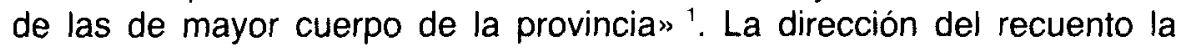
llevó personalmente el Intendente.

A esta villa se aplicaron todos los puntos de la Instrucción y Órdenes dadas por la Real Junta y desde ella salieron las disposiciones a seguir para los 326 núcleos poblados (más los 124 despoblados que fueron objeto de más de un conflicto).

1 Archivo General de Simancas (en adelante AGS). Dirección General de Rentas (DGR), primera remesa, leg. 1914. 
Se decia en la documentación general cursada:

"Siendo tan conveniente y precisa la uniformidad de las operaciones (como dirigidas a un fin) que se están ejecutando, para la averiguación de los efectos, en que pueda fundarse una sola Contribución como reglado a la Instrucción y Ordenes dadas por la Real Junta en todas las ciudades, villas y lugares y términos despoblados, y que tal vez no será bastante, el reconocimiento que los subdelegados han hecho para tener presente lo dilatado y vario de las diligencias practicadas en la villa de Ajofrín, primer pueblo de esta provincia con las precauciones hechas por la R. Junta en la vista que de ellas procedió para su aprobación, se pasa a explicar, con arreglo a lo operado y referidas advertencias, lo que parece más conveniente a su comprensión, dividiéndolo en cuadernos, de los que debe componerse el experimento de cada pueblo para que a costa de este corto trabajo, se facilite más bien la formalidad y ejecución de la Instrucción con la mayor brevedad, exactitud y conformidad" ${ }^{2}$.

La villa elegida se convertía, a la vez, en el centro de organización administrativa: desde ella se fueron cursando y repartiendo títulos a los comisionados para que lo fueran realizando en partidos y pueblos.

Tres momentos aparecen en el proceso: el primero, de formación de cuadernos relativos a los diferentes datos que se pretendian obtener; el segundo, la puesta en limpio de aquéllos, tras su verificación, o las Respuestas Particulares; el tercero, los resúmenes generales de los diferentes apartados, previa la elaboración de los individuales por pueblos.

Con respecto al primer momento, el principal, la precisión requerida era evidente.

En el cuaderno n. 1 habia que referir los autos generales. Debían constar, por un lado, "las diligencias que han de preceder para principiar la operación”.

“Todas las demás providencias de bandos, convocaciones, nombramientos de agrimensor y peritos (...) de suerte que para dar principio

\footnotetext{
${ }^{2}$ lbidem.
} 
a la formación de cada cuaderno, de los que aquí se señalarán, haya que preceder el auto y mandato del subdelegado en este cuaderno de los generales, y las demás providencias de los incidentes que ocurran, que podrán ser varios según las circunstancias de cada pueblo, siendo regla precisa el que éstas no pueden con ningún caso y motivo, alterar en nada lo prevenido en la Instrucción y Órdenes; y sí solo, dirigida para la más fácil y justificada práctica de aquellos fines, pues cuando ocurra caso nuevo, y no prevenido, deberán consultármele, para que resuelva la regla que se ha de observar o lo consulte a la Junta, cuando lo contemple necesario".

Por otro lado, las diligencias finales de la operación y la cuenta de gastos de la misma:

"Se cerrará este cuaderno con la publicación del Libro en Concejo público, certificación de los alcaldes de no haber hecho el subdelegado y dependientes gasto alguno del pueblo, y el mandato de que las diligencias se me pasen para su reconocimiento en la Contaduría principal, acompañándolas de la cuenta de gastos y salarios causados en ellas en la forma prevenida».

Sobre los demás cuadernos (hasta ocho) se señalaban las líneas de procedimiento.

Pero permanecian unas líneas generales aplicables a todos: era necesario anotar "en el acto" los posibles defectos que se encontraran porque asi se lograría "por este medio la brevedad que tanto se desea, con conocido ahorro del Real Erario".

En esta misma línea de ahorro se indicaba que no haría falta volver a repetir las relaciones que hubieran hecho los vecinos sino solamente...

“... quedar conformes las que en la realidad lo estén, o en caso de no estarlo lo que haya de diferencia, firmándolo o rubricándolo los peritos, y que de estas legitimas comprobaciones se forme el libro o libros que se manden, excusando el trabajo y gasto de los reconocimientos y cotejos, pues bastarán las notas que se insinúan para los asientos legítimos". 
Pero era claro que lo primero que habia que exigir era que los vecinos hicieran relaciones claras y precisas "con lo que al tiempo del reconocimiento será más fácil el encontrar lo que se trata de comprobar».

«Por otra parte, se advertía a los funcionarios que todo ello se había de hacer (cortejar y comprobar) conforme se fueran recibiendo las informaciones «aprovechando en este tiempo en desatar las dudas que ofreciere este cotejo, á proporción de las que fueren entregando y solicitando que no se retarden las que correspondieren a las citas de las recibidas sobre esto de los particulares, de cuya anticipada comprobación resultará no sólo abreviar mucho, pero hacer más fácilmente y sin confusión, estorbos, no celeridades, toda la operación hasta el fin..."

El mismo Intendente confesaba que, al principio, le habia faltado práctica, por lo que había dispuesto reunir las relaciones y luego cotejarlas pero que después se había dado cuenta de que el procedimiento podia ser largo y asi parecia mejor este segundo camino, de irlo haciendo todo a la vez.

«Supuesto lo antecedente, sólo queda que advertir la forma en que se deben poner las relaciones, sobre lo que nada hay que decir ni se puede adelantar a la que está establecida en Instrucción y formulario de la letra B que explica el modo de hacer el asiento de cada pieza en el libro o registro...".

Y, de nuevo, se apelaba a la necesidad de ganar tiempo:

"Como el tiempo que se ocupa (según ha mostrado la experiencia) en recoger las relaciones es casi igual al que se necesita después para perfeccionar la operación y formar los libros, es muy vital se adelante un oficial inteligente con los escribientes, que tengan por conveniente, según el vecindario al pueblo en que se haya de seguir la operación...”

Si el cotejo y la puesta en limpio de las relaciones fuese rápida podrían salir, se indicaba al Subdelegado, dos o más cuadrillas a otros tantos pueblos 
«... para que sin intermisión de una operación a otra tenga pueblos habilitados donde hacerlas, sin la detención de aguardar a perfeccionar el recogido de las relaciones que faltan".

Sin embargo, el desarrollo de las concretas operaciones fue, en muchos casos, complicado y largo. El de la villa de Ocaña (Toledo), que exponemos como ejemplo, duró desde el 8 de mayo de 1752, fecha en que se fijó el primer Edicto, hasta el 10 de julio de 1755 en que se notificó haber sacado copias de las Rentas Enajenadas de la Corona según estaba dispuesto ${ }^{3}$.

Y ello pese a la premura que se quería dar al asunto. En el mencionado Edicto de Ocaña se decía que:

«... dentro del término de ocho dias presenten (los vecinos) en la Audiencia que desde luego señalo..."

Debian éstos dar, como se sabe, razón de los componentes de su familia y de sus bienes muebles e inmuebles. Además debian detallar las cargas y censos que tuviesen las tierras y casas y se aplicaba para todo tipo de ocultación de bienes la pena de 200 ducados. Junto con el Edicto se enviaba una notificación a los Corregidores, gobernadores, alcaldes mayores, ordinarios y demás ministros en la que se les hacía responsables del cumplimiento de aquél, y si no serían responsables de los daños y perjuicios advirtiéndoles, además, que lo debían hacer en razón de su oficio, "mediante la naturaleza de la ley" sin esperar percibir maravedí alguno por ello (los alcaldes debían entregar al recibirla una nota firmada con un "cúmplase» y la certificeción de haber colocado el Edicto en sitio visible - plaza o puertas del Ayuntamiento-).

Un segundo Edicto, enviado a los diez días del primero, no cumplía otro fin que el de apremiar:

“Mediante ser noticjoso su Señoría (el Subdelegado) que el mayor número de vecinos legoș, comunidades y estado eclesiástico de la villa de

${ }^{3}$ Archivo Provisional de Toledo (APT), Catastro, libro 456. 
Ocaña no han entregado como debieran sus respectivas relaciones en el término que se les ha asignado en el primer Edicto, fijese segundo para que lo ejecuten en el de otros ocho días, bien entendidos que de no entregarlas los primeros, se pasará a la averiguación de su costa...».

$Y$ el 6 de junio un tercer Edicto "ante la contumacia en que perseveran muchos de los vecinos legos, comunidades y estado eclesiástico...". Sin embargo, el trato para unos y otros no era igual: para los legos se impondría la pena de doce ducados "con ejecución y ocho días de cárcel a cada uno de los inobedientes, aplicados a la R. Hacienda para gastos". Por "lo tocante a las mencionadas comunidades y estado eclesiástico, páseseles por el presente escribano recado de atención dándoles a entender que en el caso de no cumplir en el prefinido término (de ocho días), se dará cuenta a la superioridad..." ${ }^{4}$. El doce de junio se mandó "recado de urbanidad" a los superiores y superioras de las comunidades, asi como a los presbiteros de la villa.

Aunque venimos llamando "operación» a todo el proceso de formación del Catastro, aquella no empezaba, según se recoge en las fuentes, de una forma propiamente dicha hasta que no se tuvieran las relaciones de los vecinos seglares y eclesiásticos. En ese punto, se nombraban los funcionarios que la llevarían a cabo: reunir las relaciones, contabilizarlas, sacar los productos, pasarlas a limpio, esto era, registrarlas (de ahí los dos tipos de libros: de relaciones y de registro o de Respuestas Particulares). Fueron nombrados: un escribano, un oficial mayor, cinco amanuenses - tres que eran los mismos que habian finalizado la operación del vecino pueblo de Noblejas, dos más "por considerarse también necesarios" al ser una población mayor-, un ministro del juzgado de la villa "que asista a las diligencias que ocurran", por conocer mejor los problemas del núcleo poblado. El escribano venía también de Noblejas.

Fue entonces cuando se hizo la reunión en la Audiencia para responder a las preguntas generales, siendo convocada por el escribano de la Única (5 de julio).

Llegó para presidirla el Subdelegado (que era Justicia Mayor y Superintendente general de todas las Rentas Reales y servicios de Millones y tesorería de dicha villa y su partido). $Y$ comparecieron: los cuatro párro-

4 Ibidem. 
cos; dos regidores, uno por cada estado, nombrados por el Ayuntamiento; el síndico procurador general y el escribano del «repetido Ayuntamiento"; dos veedores "de las cosas del campo" y dos alarifes, los cuatro como "peritos nombrados por parte de la R. Hacienda y citado Ayuntamiento".

A todos ellos el subdelegado:

«... les recibió juramento, conforme se proviene en el capitulo cuarto de dicha Real Instrucción, por Dios Nuestro Señor y a una señal de la cruz en forma de derecho, quienes hicieron como se requiere y ofrecieron decir verdad...».

En ese mes de julio y en el de agosto se hicieron los reconocimientos y comprobaciones del vecindario en los que "se gastaron veintisiete días". Luego, la comprobación de los censos y cargas "para que a su tiempo (al aplicar la Unica) se tenga presente». Duró once dias, hasta el 4 de septiembre.

A continuación se procedió a la comprobación de las tierras que resultó principal punto. En octubre, en carta al máximo responsable, el Ministro Comisionado de las diligencias de Única Contribución ${ }^{5}$, preguntaba el Subdelegado si había que hacer el reconocimiento parcela por parcela, "por habérsele asegurado que sin esta conveniente diligencia (aunque dilatada) se ha practicado la operación en otros pueblos", porque era el caso que habia dueños de pequeñas piezas que no sabían precisar sus linderos y que las comunidades, capellanias, obras pias y señores "con sus criados, administradores y mayordomos estaban equivocando". En todo ello se hacia principal referencia a los bienes de forasteros.

Fue la respuesta que habria que hacerlo en los casos convenientes $y$, en especial, sobre las parcelas de forasteros, sobre las que los vecinos de Ocaña tenian en los términos colindantes y sobre aquellas que estaban imprecisas en las lindes de un término y otro.

${ }^{5}$ D. José de Oma y Haro era el máximo responsable, como Ministro Comisionado, de los partidos de Alcalá de Henares, Ocaña y Alcázar de San Juan. En tanto que el Intendente, además de su cargo general, lo era de los partidos de Toledo y Talavera. Esto explica la intervención directa del Intendente en la "primera" operación hecha en Ajofrín. 
Para ello se dio una Instrucción particular a fines de octubre de la que merecen citarse estos puntos:

2. “A este fin pasarán todos mañana a la villa de Villatobas, distante de ésta (Ocaña) tres leguas y sin perder de vista la $R$. Instrucción y Ordenes particulares, practicarán desde la madrugada del siguiente día el expresado reconocimiento de campo con asistencia indispensable de los peritos nombrados y de otros dos hombres inteligentes que diputará aquella villa en fuerza del aviso que se la dio para que con el mayor conocimiento que tienen de las heredades de sus convecinos, se identifiquen puntualmente".

3. «Siendo toda la circunferencia del pequeño término de la villa de Villatobas correspondiente a ésta, se dará principio a lo más distante para que precaviendo el rigor de los temporales, en el centro del invierno, quede lo más cercano para los intermedios serenos y capaces de trabajar, y considerando que éstos son escasos, y que el rey habrá de satisfacer íntegros los dias, se aprovecharán todos aquellos en que no lloviese ni nevase desde salir el sol hasta faltar la luz, pues aunque se verifique exceso en esto, disfrutarán en otros días de mayores descansos los oficiales ${ }^{6}$.

Con los forasteros, en general, la actuación era la siguiente: en caso de que los peritos del término no supieran exactamente lo que aquellos tenían, se enviaba una carta al lugar de origen solicitando la presencia de dos peritos (de la vecindad de la que fueran dichos forasteros) que con los del término reconocieran las propiedades. Esto restrasaba mucho la finalización de la operación; máxime, porque algunos forasteros ocultaban sus propiedades en otras villas.

Por otra parte, había que cuidar dos tipos de averiguaciones: la de parcelas sin dueños declarados y la de relaciones de vecinos sobre el terreno para comprobar "la legitimidad de sus dueños". También sucedía que el asunto podía no ser una falta de declaración de los propietarios sino desconocimiento de los peritos. Así, con fecha 2 de diciembre se decía que "por falta de puntual noticia" no se podía comprobar las heredades contenidas en "el pago y sitio que dicen de la Serna", por lo

${ }^{6}$ A.P.T., Catastro, libro 456. 
que se pedía que fueran otros dos peritos «más instruídos de dicho pago".

A los eclesiásticos que ocultaban se les mandó la oportuna notificación para que presentaran los motivos que habian tenido para ocultar, lo reconociesen legitimamente y "acrecentaran sus relaciones" con dichas posesiones.

A finales de diciembre la Justicia de Villatobas no había logrado reunir a los propietarios de bienes de Ocaña y se excusaba diciendo que unos estaban ausentes,

"... otros porque ignoran quiénes sean y otros por ser doncellas, de corta y crecida edad, y viudas honradas que por sus circunstancias con dificultad se pondrian en camino de comparecer en esa Audiencia".

Asi debieron seguir las cosas, luchando los funcionarios contra las dificultades y las puestas en limpio, porque los Libros se acabaron de hacer a fines de junio.

El 26 de junio de 1753 fue el día señalado para que se diera lectura a los mismos en la plaza pública "con asistencia de los Capitulares del Ayuntamiento", indicando que el acto empezaría desde el día 27 "a las siete y por la tarde a las cuatro". Fueron leídos los días 27,28 y 29 «del que no resultó quejarse agraviado vecino alguno de los que concurrieron, ni dado a entender haberse ocultado ningunos bienes".

Luego se procedió a elaborar los resúmenes de los productos y alquileres, de las clases de tierra, el número de medidas, etc. Quedando para el final la recopilación de las copias de los privilegios de las Rentas Enajenadas de la Corona.

La masa de datos resultante, como se comprenderá, fue copiosa y los gastos de elaboración elevados según se desprende de la documentación consultada. $Y$ ello pese a las estrictas disposiciones de los Intendentes que pretendían eliminar todo lo que se considera superfluo.

Sirva como muestra que los gastos finales de la operación de 23 pueblos de la provincia de Toledo (el siete por ciento del total -325-) ascendieron a 265.938 rs. vn., y ello sin contar con otros derivados, en 
muchos casos, de la prolongación temporal de las fases segunda y tercera del proceso (puesta en limpio y resúmenes) por acumulación del trabajo amanuense. Otros gastos generales, como los de encuadernación, quedaban añadidos sólo al final: en 1756 se calculaba que la encuadernación en pergamino de los legajos de la Única provincia de Toledo iba a costar entre 3.500 y 4.000 rs. ?

Pero concurrían muchos factores. En la correspondencia que las diversas provincias debían enviar a la Junta se registran frecuentes notas sobre los empleados: o contra éstos o de éstos sobre el trabajo y la escasez de sueldos percibidos.

Hubo, hay que decir, una general lentitud en el proceso de elaboración que no tenía por qué venir de la entrega de las relaciones de los vecinos; el 4 de enero de 1752, se decía que en la provincia de Toledo, los partidos de Toledo y Talavera habían finalizado 14 operaciones de los pueblos pero quedaban 190 por acabar.

Y en los de Ocaña, Alcalá y Alcázar se habian hecho sólo cuatro y quedaban 126 por completar $^{8}$.

La misma operación de la cuidad de Toledo comenzó el 9 de junio de 1751 y concluyó el 28 de octubre de $1753^{\circ}$.

$Y$ en cuanto al proceso burocrático de reunir, copiar etc. en 11 de enero de 1757 quedaban todavía 123 operaciones de pueblos por copiar ${ }^{10}$. En la provincia de Extremadura todavía el asunto fue más lento porque en $1 .^{\circ}$ de abril de 1760 , de las 386 operaciones quedaban por terminar todavía 111 (se consideraba "completa" o "finalizada" una operación cuando quedaba puesta en limpio con todos los productos o lista para la aplicación de la Única) ${ }^{11}$.

Ya para 1752 se pensaba en Toledo que la elaboración iba lenta. Llegó al partido de Toledo una resolución de la Junta (11 de enero) según la cual se exigía a los escribanos de la Audiencia

\footnotetext{
7 A.G.S., D.G.R., 1 remesa, leg. 1917.

8 Ibidem, leg. 1914.

9 Ibidem, leg. 1916.

${ }^{10}$ Ibidem, leg. 1917.

11 Ibidem, leg. 1873.
} 
“... a que trabajen, y que se empleen en todos los asuntos de las operaciones, como cualquiera de los oficiales o escribientes" ${ }^{12}$.

A veces aparecen quejas de que los comisionados no se apresuraban a acabar para seguir cobrando el sueldo: así, se escribía desde Mejorada (Talavera) «que los comisionados no caminan todos a un fin, sino a que dure la dependencia, particularmente los de aquel partido (Talavera), siendo los escribanos los que tienen la mayor culpa por sus malas prendas". Se decía que la operación en dicho pueblo de 240 vecinos había costado 15.000 rs. y que se podía haber hecho por menos de la tercera parte «de este dinero y tiempo».

Se acusaba al Subdelegado y al escribano. De aquel se escribía: "y que de esto es la causa ser el subdelegado un pobre hombre que tiene por oficial a un sobrino que hace lo que le da la gana, y los escribientes, que son de su parte, lo mismo". Y del escribano:

«... que el escribano que lo es del número de la villa de Talavera y se llama Pedro Carrero, es hombre de mala fama e intención, y ha puesto cuatro escribientes: los dos sobrinos suyos y los otros dos escribientes de su oficio, quitando para esto al mejor que tenía la Audiencia" ${ }^{13}$.

En Tornavacas (Extremadura) las quejas se centraban en el Subdelegado "por su genio díscolo y las inquietudes que ocasiona en aquel pueblo". Y otra vez los dispendios: se escribía que la operación en ese pueblo, por culpa del mencionado Subdelegado, había costado 37.000 rs., en tanto que en Cabezuelas, villa inmediata y de iguales circunstancias, había costado 5.000 , "cuya diferencia es digna de notarse» ${ }^{14}$.

En la Contaduría de La Coruña se recibió una queja en 1759 contra los administradores generales de Vigo, Redondela, Pontevedra, Marin y otros puertos "sobre sus crecidos caudales y ninguno con que entraron

12 Ibidem, leg. 1915.

13 Ibidem.

14 Ibidem, leg. 1873. 
en el sercivio", y se añadía: "para que se tenga presente el tiempo de establecimiento de una sola Contribución» ${ }^{15}$.

Tal tipo de quejas podrían multiplicarse. En Toledo hubo pueblos que se opusieron a los comisionados por ser

«... sujetos tan indignos como faltos de inteligencia (...) por lo que se miraba con desprecio su dependencia con la Unica”" ${ }^{16}$.

Pero permanecía el problema de la lentitud en el finalizar, con notable gasto para el Erario.

El Intendente de la provincia dispuso, en 1752, que oficiales y escribientes trabajaran en la oficina cuatro horas por la mañana y cuatro por la tarde "de modo que las primeras cumplan a las doce del mediodia y las restantes a las diez de la noche, dándoles (si Ud. lo tiene a bien) un vidrio de agua durante la estación de los calores por la tarde mediante el corto desperdicio que esto pueda originar a la R. Hacienda..." Se preveía darles descanso los domingos, jueves por la tarde "y las de los días clásicos" " $"$.

Y en 1754, la Junta todavia precisó más:

«... ha resuelto que en las Contadurías de las 22 provincias se trabaje por los oficiales de ellas ocho horas cada dia sin más excepción que el primero y segundo (día) de las Pascuas, en que no se comprenden los domingos y demás festividades, pues en éstos se debe trabajar" ${ }^{18}$.

Debía haber muchas ausencias al trabajo: bastantes documentos hacen referencia a solicitudes de permisos aduciendo diversos motivos, siendo los más corrientes las enfermedades de familiares, o la necesidad de ayudar en las faenas del campo, como un comisionado de la ciudad

${ }^{15}$ Ibidem, leg. 1877.

${ }^{16}$ Ibidem, leg. 1914.

17 Ibidem, leg. 1915.

18 Ibidem, leg. 1873. 
de Toledo que pidió licencia de doce días para ir al cercano pueblo de Polán a la recolección de la uva ${ }^{19}$.

En 1756 resolvió la oficina de Única de la ciudad de Toledo que se les descontase de sus sueldos los días en que los dependientes faltasen sin legítimo motivo, y que se llevara un libro.

«... donde diariamente se apunten la asistencia de todos y sus respectivas faltas para que sirva de documento que gobierne los descuentos ${ }^{20}$.

Cierto era que, por contra, las oficinas apelaban a la escasez de personal y de sueldos. En 1751 se exponía en Toledo «el crecido gasto de caballerías para conducir los escribientes al reconocimiento de campo, a que no les sufragaba su corto sueldo". Y la respuesta fue "que por estar negado a otros de igual clase no se les podía conceder» ${ }^{21}$. En 1753 solicitaron dichos escribientes cobrar nueve rs. diarios - cobraban ocho rs. - y les fue denegada la petición.

Muchos documentos de Galicia señalan lo mismo: los oficiales y escribientes se quejaban del gran trabajo, por "ser pocos", y de los bajos sueldos ${ }^{22}$.

$Y$ en Extremadura se anotaba, en los años finales de la década de los 50 , que no solamente se habian retirado empleados de las oficinas sino que se habian rebajado los sueldos. Badajoz decía a la Junta en 1760 que si los trabajos iban retrasados era porque en la oficina no habia más que dos dependientes y un Comisionado ya muy mayor, con más de 40 años de servicios para Hacienda (mencionándose, incluso, el «deplorable estado" de éste ${ }^{23}$.

En cambio, no hay en estos años demasiadas cartas sobre la oposición de los futuros contribuyentes, quizás porque aquélla había finali-

\footnotetext{
19 Ibidem, leg. 1917.

20 Ibidem, leg. id.

21 Ibidem, leg. 1914.

22 Ibidem, leg. 1877.

${ }^{23}$ Ibidem, leg. 1873.
} 
zado con la entrega de sus relaciones. Con todo, en 1755 se anotaba que los productos que se estaban señalando al margen de las parcelas (de acuerdo con la extensión, calidad de la tierra y precio de los frutos) podían no valer "por la decadencia notable que la adversidad continuada de los años anteriores ha ocasionado en los productos de muchas partes de las clases", y que aunque esto en la tierra podía paliarse aceptando

“... un año de esterilidad y otro de riqueza, sin embargo, no sucede así en los ganados, sus diezmos y las colmenas".

La preocupación de la $R$. Junta era que se redujeran los excesivos gastos de escritorio y que se mantuvieran solamente los empleados indispensables, exigiéndoles la asistencia... y que acabara cuanto antes lo emprendido. Así se pasó de tener que dar cuenta cada mes a los Intendentes de cómo marchaban las operaciones de los pueblos a tener que avisar semanalmente desde las oficinas a Madrid el número de hojas que se habían copiado ${ }^{24}$.

Y todo ello porque la elaboración del Catastro había resultado una "fuente de empleo y de salarios" a cuenta de Hacienda.

La petición de solicitudes para incorporarse a la nueva labor fueron muchas como recoge la documentación estudiada. Otras veces, fue un medio de "compensar" otras situaciones. Tal fue el caso de D. José de Oma y Haro, máximo responsable de los partidos de Alcalá, Ocaña y Alcázar de San Juan. Le fue otorgado este cargo con un sueldo de 26.000 rs. pero en la instancia que había presentado solicitaba otra cosa:

“D. José de Oma y Haro, del Consejo de V.M. en el Tribunal de Cuentas de la Contaduria mayor de Cuentas, dice: ha diez y seis años que está sirvierido a V.M., los nueve de cadete en las R. Guardias de Infantería española y los siete en el ejercicio de la plaza de Ministro del Tribunal, siempre sin sueldo, por to que ha consumido su patrimonio en su decente manutención, y no teniendo otro recurso para continuar, como le dicta su amor y celo, que el de la piedad de V.M.

Suplica a V.M. rendidamente se sirva concederle, o el sueldo o el medio sueldo de la dotación de su empleo, o conferirle interinamente,

${ }^{24}$ Ibidem, leg. 1877. 
ya hasta que entre en plaza de número el empleo de gobernador del campo de Madrid que está vacante por fallecimiento de D. Pedro Molina» ${ }^{25}$.

Pero a favor de los elaboradores del Catastro hay que hacer referencia a las múltiples dificultades concretas que tuvieron que solventar para dar unidad "a lo dispuesto". En la ciudad de Toledo se decía que como no había padrón de vecinos para saber quiénes entregaban las relaciones, hubo que proceder a solicitar las "Matrículas anuales de las parroquias para el cumplimiento de la Iglesia». La misma recopilación del censo demográfico debió ser dificultosa, de modo que "para abreviar el tiempo con ahorro de salarios a la R. Hacienda» se propuso que los sacristanes de las respectivas parroquias ayudasen al reconocimiento de las casas y comprobación de las familias» ${ }^{26}$.

La misma cuestión del apartado de «lo personal» (salarios) provocó toda una serie de dudas. Baste indicar que los mancebos de las tiendas de comercio (en Toledo) tenían cuatro tipos de forma de cobro de salario: - los que cobraban un fijo diario más la manutención; - lo que cobraban un salario sin manutención; - los que recibian manutención y vestido, y los que recibian sólo manutención. Igual sucedía en Extremadura con los pastores, ayudadores y zagales; o con los jornaleros bajo cuyo concepto se incluian tres clases: los del campo, en artes mecánicas y las gentes de librea; por lo que la duda era si había que aplicarles 120 días anuales o 180 de trabajo ${ }^{27}$.

O la pregunta de quiénes eran los receptores o mayordomos seglares de las Hermandades y Cofradías porque ellos tenían la relación de bienes de las mismas. O la recogida de las copias de los oficios de la ciudad vendidos por la Corona cuyos propietarios no residian en ella, y eran los más.

En 1760 todavía quedaban provincias por finalizar su Catastro y no había entrado en aplicación la Única. Se seguía con las Rentas Provinciales en manos de Hacienda y ésta tan sólo había introducido la innovación de suprimir ( 7 de junio 1758) la renta de Servicio y Montazgo que

${ }^{25}$ Ibidem, leg. 1914.

26 A.P.T., Catastro, libro 716.

27 A.G.S., DGR, 1 remesa, leg. 1873. 
le pertenecía a cambio de un equivalente que consistía en cobrar la salida de las lanas de los reinos de acuerdo con un canon ${ }^{28}$.

El esfuerzo de la elaboración de un Catastro "desde arriba" no se volvería a repetir. Las Comprobaciones de 1760 y 1770 hubieron de hacerse a cargo de los presupuestos municipales por no poder Hacienda hacer frente a los gastos. $Y$ ahí empezaron las contradiciones del reformismo ilustrado porque la Única Contribución no entraría nunca en vigor.

${ }^{28}$ A.H.N., Sec. Hac., libro 8021. 